\title{
SOUND UND BILD: DIE AUdIO/VISUELLE ÖKONOMIE musikalischer Performance ${ }^{1}$
}

\author{
Philip Auslander
}

Eine gegenwärtige Diskussion im Bereich der Computermusik berührt eine fundamentale Frage hinsichtlich der audiovisuellen Ökonomie der musikalischen Performance. Von den Zuschauern musikalischer Aufführungen, die mit einem traditionellen Instrumentarium arbeiten, wird angenommen, dass sie die zugrundeliegenden Kausalzusammenhänge zwischen dem, was sie sehen und dem, was sie hören, verstehen (wenn ich z.B. sehe, wie ein Musiker die Taste eines Pianos drückt oder in das Klavier hineingreift, verstehe ich den folgenden Klang als Resultat der Handlung, die ich zuvor beobachtet habe). Anders verhält es sich mit dem Publikum einer Musik, für deren $\mathrm{Er}$ zeugung verhältnismäßig ungewohnte, digitale Geräte wie unterschiedliche MIDI-Interfaces oder Laptops als Instrumente benutzt werden. Bei diesen Zuschauern kann nicht davon ausgegangen werden, dass sie das Verhältnis zwischen der Handlung des Performers und den daraus resultierenden Klängen verstehen (mit Ausnahme jener natürlich, die mit den jeweiligen Technologien und angewandten Techniken gänzlich vertraut sind). Caleb Stuart führt dieses wie folgt aus:

»With the laptop, there is no [cause-and-effect] connection. From the point of view of the audience, the computer is an inanimate object; it sits there while the performer acts surreptitiously behind the screen. [...] The audience in general does not know exactly what it is that the performer is doing and most do not know how the sound is produced or with what« (Stuart 2003: 61).

Diese maskierte Art der Klangproduktion ist eine Herausforderung für das Verständnis der Beziehung zwischen Musiker und Publikum, welches ich die

1 Dieser Artikel erscheint in einer englischsprachigen Version in The Oxford Handbook of New Audiovisual Aesthetics. Hg. v. Claudia Gorbman, John Richardson und Carol Vernallis (Oxford University Press). Ich danke Oxford University Press für die Genehmigung, die deutsche Übersetzung hier abzudrucken. Ins Deutsche übertragen wurde dieser Artikel von Yvonne Thieré, bei der ich mich herzlich bedanke. 
»traditionalistische« Sichtweise nennen werde. Einige Aspekte dieses Standpunktes werden von W. Andrew Schloss skizziert, wenn er schreibt: »This relationship is based on many factors, most significantly on trust, and also on the audience understanding what the performer is doing on stage « (Schloss 2003: 239; Hervorhebung im Original). Zwar ist man sich in der Diskussion um Computermusik darüber einig, dass neue Musiktechnologien die traditionelle Beziehung von Musiker und Publikum herausfordern, jedoch wird diese Herausforderung von den einzelnen Seiten ganz unterschiedlich betrachtet. Schloss repräsentiert die traditionalistische Seite: »A visual component is essential to the audience, such that there is a visual display of input parameters/gestures« (ebd.: 242). Diese sichtbaren Handlungen verdeutlichen das Wesen der Kausalität innerhalb einer Performance. Stuart hingegen argumentiert:

»The performativity of the music is to be found in the act of listening and the performance of the audience in relationship to the sound they hear. There is no need then for us to see a performer physically interacting with an instrument to engage in this aural performativity: we need only listen and engage in the act of listening « (Stuart 2003: 64).

Obwohl diese Debatte von dem beschleunigenden Einfall digitaler Technologien in die Live-Performance von Musik angetrieben wird, sind die zugrunde liegenden Fragen keine neuen. Die derzeitige Debatte ist Teil der seit längerem geführten Diskussion über das, was Pierre Schaeffer, der eine zentrale Rolle für die in den 1940er Jahren beginnende Entwicklung der Musique Concrète einnahm, »acousmatic sound « nannte und was von Jonathan Sterne als »sounds that one hears without seeing their source definiert wurde. ${ }^{2}$ Die Idee, dass die auditiven und visuellen Dimensionen der musikalischen Performance voneinander unterscheidbare »tracks« sind, und die Frage danach, wie die Beziehung dieser Tracks verstanden und in der Performance konfiguriert werden sollten, ist in ganz unterschiedlichen Kontexten aufgekommen, spätestens seit es die Aufnahmetechnik ermöglicht hat, einen Klang unabhängig vom Visuellen zu erfahren (vgl. Laing 1991: 1-9). Die Auffassung dieses Verhältnisses, die das Denken des 20. Jahrhunderts bestimmt hat und weiterhin einen starken Einfluss ausübt, reiht sich in die gut dokumentierte westliche Bevorzugung des Sehsinnes ein. ${ }^{3}$

2 Sterne (2003: 20-22) gibt eine kurze Zusammenfassung der Diskussion dieses Konzeptes und seiner Auswirkungen.

3 Die Literaturlage zum Okularzentrismus der westlichen Kultur ist gewaltig. Eine klassische Untersuchung von beidem, dem Okularzentrismus und seiner kritischen Abhandlungen, stammt von Martin Jay (1994). 
Die traditionalistische Sichtweise bestätigt die Wichtigkeit der »visual communication of musically relevant information «, beschränkt diese Kategorie aber generell auf Gesten und Gesichtsausdrücke der Musiker während sie auftreten (Schutz 2008: 86). ${ }^{4}$ Für Schloss bedingt die musikalische Performance die Zurschaustellung des Aufwandes, den ein Musiker zugunsten des Publikums betreibt und der die Aufwendung physischer Kraft sowie die moralische Hingabe offenlegt »to what one is doing " (Schloss 2003: 240). Stan Godlovitch, der ein detaillierteres traditionalistisches Model der musikalischen Performance liefert, nennt in einer Auflistung von Bedingungen, die ein Ereignis zu erfüllen habe, um als musikalische Performance zu gelten, dass das Ereignis »the immediate output of some musical instrument « sei (was Computer nicht mit einbezieht, Godlovitch 1998: 101), »the exercise of skilled activity « einschließt und »the outcome of appropriately creditworthy physical skill« sei (Godlovitch 1998: 49). Sowohl Schloss als auch Godlovitch machen die essentiellen Aspekte musikalischer Performance an Dingen fest, die durch Klang allein nicht direkt zu begreifen sind. Godlovitch fechtet die Idee an, dass Hörer, die bei Konzerten ihre Augen schlieBen, alles erführen »that is musically significant «, denn »musical sound alone is not sufficient for performance (ebd.: 14f.). Die Anstrengung oder das Geschick des Musikers könne man nicht hören; man müsse die »bulging veins in the neck of the trumpeter blasting a high $\mathrm{C}$ « sehen können, um den Einsatz vollständig würdigen zu können (Schloss 2003: 240). Zudem könne man sich nicht sicher sein, dass der Klang eines Musikers ein direktes Produkt seines Könnens ist, wenn man ihn nicht bei der Produktion des Klanges beobachtet habe. Hierbei ist zu bemerken, dass das Musik-Publikum in den meisten Fällen wahrscheinlich keinen sehr detailgenauen Sinn für instrumentale Kausalzusammenhänge hat. Für Blasinstrumente bemerkt Michael Schutz (2008: 101): „Changing pitches requires complex interactions between embouchure and fingertips that are far from transparent to audiences «. ${ }^{5}$ Folglich ist die traditionalistische Betonung der sichtbaren Kausalität in musikalischen Darbietungen am besten als ideologisch zu verstehen. In den meisten Fällen versteht das Publikum nicht wirklich, wie der Klang im Einzelnen produziert wird. Es möchte aber dennoch glauben, dass es das kann.

4 Wenngleich ich die Phrase »musically relevant information« von Schutz übernommen habe, halte ich ihn nicht für einen Repräsentanten der traditionalistischen Position.

5 Theodore Gracyk (1997: 145) kritisiert Godlovitch mit einem ähnlichen Argument. 
Aus traditionalistischer Sicht ist die Integrität einer musikalischen Performance von allem bedroht, was verhindert, dass das Publikum den Musiker als qualifizierten kausalen Handelnden der Performance erkennt, einschließlich Tonaufnahmen, ablenkender Spektakel oder des Gebrauchs digitaler Technologien, die die Zusammenhänge von Ursache und Wirkung undurchsichtig machen. ${ }^{6}$ Godlovitch beispielsweise argumentiert, dass »whatever we hear on a recording is not itself sufficient to ground judgments of the player's real role and true merit«, denn aus der Aufnahme selbst kann der Hörer nicht schließen, wie diese genau angefertigt wurde (Godlovitch 1998: 26). Selbst wenn man von der Performance auf einem Tonträger annimmt, dass sie Virtuosität repräsentiert, oder wenn man Dinge hört, die den Einsatz und die Hingabe des Musikers suggerieren (z. B. Glenn Goulds berühmte Zwischenrufe oder das > Jaulen< und Aufschreien von Gitarrist Alvin Lee auf der Ten Years After-Aufnahme von »Boogie On«), hätte man unmittelbar die Performance beobachten müssen, um schlussfolgern zu können, dass die aufgenommenen Klänge tatsächlich bedeuten, was sie zu bedeuten scheinen.

Wenn eine neue Technologie oder ein Medienformat, einschließlich Radio, Tonaufnahme, Musikvideo und der Einsatz von Computern in einer Performance, droht, diese Art der visuellen Verifizierung in Frage zu stellen, beunruhigt dies die Traditionalisten. Diese Sorge teilen die musikalischen Traditionalisten mit den Theoretikern der Akusmatik, die, wie Sterne ausführt, annehmen »that face-to-face communication and bodily presence are the yardsticks by which to measure all communicative activity« und fürchten, bestimmte Technologien und Performance-Praktiken de-kontextualisierten den »sound from its sproper interpersonal context « (Sterne 2003: 20f.). In meinem Buch Liveness: Performance in a Mediatized Culture stelle ich die Hypothese auf, dass die Kategorie der »Liveness « erstmals gebraucht wurde, um einige Performances von anderen zu unterscheiden (das Oxford English Dictionary datiert die früheste Verwendung dieser Art auf 1934) als Reaktion auf eine solche Angst, die zu dieser Zeit durch die Dominanz des Radios aufkam. Frühe Technologien der Schallaufzeichnung haben die Unterscheidung zwischen live und aufgenommen nicht problematisiert: Spielte man eine Schallplatte auf einem Grammophon ab, wusste man ganz genau, was man gerade tat und es gab keinerlei Möglichkeit, das Hören einer Aufzeichnung mit dem Erleben eines Konzertes zu verwechseln. Das

6 Aus rhetorischen Gründen behandele ich den traditionalistischen Standpunkt als wäre er monolithisch. Das ist er selbstverständlich nicht. Die Befürworter traditionalistischer Werte würden der von mir postulierten Orthodoxie sicherlich in unterschiedlichem Grad zustimmen. 
Radio ist jedoch ein blindes Medium, dass es unmöglich macht, den Ursprung der wahrgenommenen Klänge zu verifizieren. Zur Lösung dieser Krise entwarf man die Etiketten »live« und »aufgezeichnet«. Hiermit konnten im Diskurs Live-Übertragungen von aufgezeichnetem Material unterschieden werden, was den Radiohörern folglich erlaubte zu wissen, ob sie entweder die unmittelbaren Resultate der qualifiziert agierenden Musiker hörten oder eine Aufnahme (Auslander 2008: 59f.).

In der aktuellen Literatur manifestiert sich diese Sorge u.a. in Godlovitchs (1998: 69f.) Bedenken, dass digitale Technologien die »echten « Musikinstrumente und Fähigkeiten letzten Endes ersetzen werden. Auch Schloss (2003: 239f.) äußert Befürchtungen, dass die Undurchschaubarkeit der Ursache-Wirkung-Verhältnisse in Performances mit Interfaces, mit denen die meisten Hörer nicht vertraut sind, die Hörer entfremden wird. Ähnliches formulieren auch Thompson, Graham und Russo, wonach eine Reihe von Phänomenen wie die Bildsprache des Musikvideos, spektakuläre Popmusik-Performances und die Luftgitarre die Aufmerksamkeit des Publikums von jenen visuellen Informationen verdrängen bzw. ablenken, die »musikalisch relevant « sind (Thompson/Graham/Russo 2005: 221-224).

Ein wachsender Teil der Rezeptions- und Wahrnehmungsforschung von Musik in der experimentellen Psychologie stützt in gewisser Weise die traditionalistische Sichtweise und ihre Bedenken: Studien zur Klangwahrnehmung deuten darauf hin, dass diese insofern multimodal ist, als dass nicht nur der auditive, sondern auch der visuelle Sinn dazu beiträgt und dass das Gehirn immer danach strebt, zwischen einem gehörten Klang und einer sichtbaren Quelle kausale Zusammenhänge herzustellen (Schutz 2008: 85). Experimentelle Studien zu musikalischen Performances zeigen, dass die visuelle Dimensionen deutlich beeinflusst, was wir hören. ${ }^{7}$ Die Werte jedoch, die mit den zwei Sinnesmodalitäten - Hören und Sehen - bei einer musikalischen Erfahrung verbunden werden, und die hierarchischen Beziehungen zwischen beiden sind kein Produkt menschlicher Biologie, sondern kulturellen Ursprungs.

Obwohl jede Musikwahrnehmung multimodal ist, kommt es nur in manchen kulturellen Kontexten vor, dass Publika die Strukturierung der Beziehung zwischen den Modalitäten verlangen, um die Anstrengungen des Musikers offenzulegen und seine Vermittlungshandlung zu bestätigen. Obwohl solch eine Transparenz für die Traditionalisten zwingend ist, scheint es doch offensichtlich, dass beispielsweise die Mehrheit des Publikums der Black Eyed Peas oder Lady Gagas in keinster Weise besorgt ist, dass deren Ge-

7 Ein Überblick findet sich bei Schutz (2008). Eine detailliertere Darstellung verschiedener Experimente geben Thompson/Graham/Russo (2005: 210-220). 
brauch digitaler Technologien zur Produktion instrumentaler und vokaler Klänge bei einem Auftritt es nicht ohne Weiteres ermöglicht, die Zusammenhänge von Ursache und Wirkung zwischen musikalischen Klängen und den nötigen Mitteln zu deren Produktion zu identifizieren. Godlovitch basiert sein Model zur musikalischen Performance ausdrücklich auf dem Beispiel klassischer Konzertsolisten. Schloss lässt eine Bestimmung des Musikgenres vermissen, mit dem er sich hauptsächlich beschäftigt, verbindet in seiner eigenen Karriere als Percussionist und Computermusiker jedoch Jazz, Rock, Latin und zeitgenössische Kompositionen (University of Victoria o.J.). Es ist ebenso plausibel, dass das Publikum klassischer Musik und zeitgenössischer Kompositionen an traditionellen Werten festhält, dass sie sehen und verstehen wollen, wie die Musik, die sie hören, produziert wurde, wie es verständlich ist, dass das Publikum von Pop- und Tanzmusik, die mit digitaler Technologie produziert wird, diese Werte nicht zwingend teilt. Auf der anderen Seite müssen Musiker und ihr Publikum aus letztgenannten Kontexten traditionalistische Werte auch nicht zwangsläufig verwerfen. Das Verlangen, die transparenten Beziehung zwischen Musikern und Publikum aufrecht zu erhalten, wie die Traditionalisten es sich vorstellen, ist nicht auf Kunstmusik beschränkt; es ist ebenso zentral für die Ideologien der meisten Formen von Rock, Jazz, Blues, Country, Folk und anderen Genres der populären Musik. In einer Arbeit, die auf ethnografischen Studien über Berliner Club DJs basiert, beobachtet Mark J. Butler:

»To the extent that they are expected to convey liveness in performance, musicians must also communicate connections between physical gestures and resultant sounds to their audiences. This is especially important in an electronic dance music context, in which many of the musician's interactions with interfaces may be invisible, and the unfamiliarity of the instruments renders their performance techniques gesturally opaque to most audience members « (Butler o.J.).

Aus diesem Grund streben die DJs in ihrer Verwendung von Technologie nach »Leserlichkeit« (»legibility«). In Liveness diskutiere ich, wie die Authentizitätsideologie des Rock dazu führt, dass das Publikum akzeptiert, dass die Musiker auf der Bühne oder einer Aufnahme, die verantwortlichen Akteure für die Klänge sind, die es hört (parallel zu Godlovitchs Analyse klassischer Musik). Jeder Zweifel an dieser Tatsache (an dem Einsatz aufgenommenen Materials in Konzerten [z.B. Lippensynchronisation] oder von Studiomusikern bei der Einspielung beispielsweise) kann die betreffende Musik als inauthentisch diskreditieren. Die Authentizität des Klangs an sich kann aber nicht nachgeprüft werden - das Publikum kann eine solche Überprüfung nur durchführen, indem es die Musiker beobachtet und aus dem 
erkennbaren Verhältnis zwischen ihren Handlungen und den produzierten Klängen Schlüsse zieht (Auslander 2008: 91).

Nichtsdestoweniger können Performer, die in den von traditionellen Werten beherrschten Genrekontexten agieren, diese Werte hin und wieder herausfordern, indem sie die Beziehungen zwischen den auditiven und visuellen Aspekten der musikalischen Darbietung auf eine Art und Weise manipulieren, die gegen den traditionellen (oder traditionalistischen) Strich geht. Im verbleibenden Teil dieses Artikels werde ich mir eine solche Performancepraxis anschauen: den Einsatz von Light-Shows in Psychedelic Rock-Konzerten.

\section{Liquid Light}

Eine sehr direkte Herausforderung für die traditionalistischen Überzeugungen stellte die Praxis einer Reihe prominenter Psychedelic Rock-Bands der 1960er dar, darunter The Doors, Jefferson Airplane und anderer, die Teile ihrer Konzerte im Dunkeln darboten und damit die Art und Weise reproduzierten suchten, wie Menschen Platten hören. Sie deuteten damit an, dass musikalischer Klang autark ist und keine visuelle Verifizierung erfordert (Auslander 2006: 18). Jefferson Airplane erachteten den Punkt als wichtig genug, um den Song »Turn Out The Light« auf ihrem Album Bless Its Pointed Little Head zu veröffentlichen, eine Liveaufnahme, die 1968 im Fillmore East und Fillmore West entstand. Auf der Aufnahme hört man Mitglieder der Gruppe, die inständig darum bitten, die Bühnenbeleuchtung zu dimmen; ihr Flehen wird zu einer improvisierten Instrumentalnummer mit einem abgedroschenen Country-Feeling. Bei sorgfältigem Hinhören offenbart sich jedoch, dass die Band die Performance in der Dunkelheit nicht als Selbstzweck ansieht. Vielmehr geht es innen darum, dass ihr Publikum auch in der Lage ist, die begleitende Light Show zu sehen.

Während der psychedelischen Ära waren Light Shows ein zentraler Teil von Rockkonzerten. Jefferson Airplane engagierten den von San Francisco aus operierenden Glenn McKay. Seine Headlights genannte Truppe, die häufig im Fillmore Auditorium, dem späteren Fillmore West, performt hatte, sorgte von 1967 bis 1972 exklusiv für die Light Show der Band (Tamarkin 2003: 146). Das Fillmore East in New York City hatte einer feste Gruppe von Lichtkünstlern unter der Leitung Joshua Whites, die als die Joshua Light 
Show bekannt wurde. ${ }^{8}$ Jefferson Airplane wollten ganz offensichtlich, dass ihr Publikum in den Genuss ihres Zusammenspiels mit den Headlights kommt, aber ihre Bitte um Dunkelheit hatte noch andere Gründe.

Als eine Performance, zu der zwei Gruppen von Künstlern mit unterschiedlichen Medien etwas beitragen, kann man das Rockkonzert mit Light Show als ein Beispiel musikalischer Multimedialität (»instance of musical multimedia«, IMM) analysieren. Nicholas Cook hat hierfür einen theoretischen Rahmen geliefert. Cook unterscheidet Typen multimedialer Events hinsichtlich des Charakters der Verhältnisse zwischen den verschiedenen Medien. Diese Verhältnisse können dreierlei Art sein: Konformität (»conformance $«)$, Komplementierung (»complementation«) und Wettbewerb (»contest «). Konformität beschreibt Situationen, in denen andere Medien mit der Musik übereinstimmen (und umgekehrt); Komplementierung beschreibt Situationen, in denen Musik und andere Medien einander vervollständigen, um einen Gesamteindruck zu formen; Wettbewerb beschreibt Situationen, in denen Musik und andere Medien miteinander in Konflikt oder Konkurrenz stehen. ${ }^{9}$ Wie Cook richtig vorschlägt, sind diese Kategorien möglichst nicht als diskrete zu behandeln: »A more sensitive application will distinguish between the different roles played by different media within any IMM and will categorize the relative preponderance of conformance, complementation, and contest « (Cook 2001: 106).

Konzerte mit psychedelischen Light Shows waren komplexe und facettenreiche Formen musikalischer Multimedialität. Beschreibungen weisen häufig darauf hin, dass die Bewegung der Lichter, Formen, Farben und Bilder »were based on the underlying rhythm of the music « und einen »direct link between the visual and aural effect « etablierten (Whiteley 1992: 28f.). Auf dieser Basis können die Konzerte als Fälle von Konformität der Lichter mit dem Rhythmus oder der thematischen Struktur der Musik beschrieben werden. David Snyder hingegen, der unter dem Namen Revelation Lights

8 Vgl. Zinman 2008: 17-21; Pouncey 2005a: 155-162 und 2005b: 163-178. Für Darstellungen, die die Joshua Light Show und verwandte Phänomene in einen kulturellen Kontext einordnen vgl. Iles 2005: 67-83 und Grunenberg 2005: 21-35.

9 Kay Dickinson (2007: 15) kritisiert Analysen, in denen audiovisuelle Formate behandelt werden »as a parade with one leader [rather than] a thoroughfare with two-way traffic «. In diesem Kontext sollte man darauf hinweisen, dass obwohl Cooks Vorstellung von Multimedialität von klaren Unterscheidungen zwischen Medien und den Sinnen, auf die sie wirken, abhängt, nur die Kategorie der Konformität »führende« (»leader«) und »folgende« (»follower«) Medien umfasst. Wettbewerb ist eher ein Zustand von Spannung zwischen den Medien, die ein IMM bilden, als dass eines der Medien das andere dominieren würde, während Komplementierung überhaupt keine hierarchische Beziehungen zwischen den Medien impliziert. 
auftrat, beharrt darauf, er habe nie gewollt, dass das Publikum dachte, die Lichter folgten der Musik, sondern, dass Lichter und Musik simultane Interpretationen der Komposition darstellten (vgl. den Videoclip »Virgil Fox Heavy Organ«). So verstanden, könnte das Konzert mit einer Light Show das sein, was Cook als »triadic conformance « beschreibt: Musik und andere Medien stehen mit einer dritten Entität in wechselseitiger Konformität zueinander (in diesem Fall die musikalische Komposition) (Cook 2001: 101).

Oftmals wird zudem behauptet, Psychedelic Rock und Light Shows hätten zusammen danach gestrebt, das Erleben eines LSD-Trips zu steigern oder zu simulieren. McKay erklärt: »Even if you weren't tripping, [the light show] gave you another trip« (zit. n. Hamlin 1999). Im Stück»Turn Out The Lights « droht ein Mitglied von Jefferson Airplane der Bühnencrew im Scherz, er schicke »Owsley to get you«, wenn die Lichter nicht gedimmt würden eine Anspielung auf Owsley Stanley, der zu dieser Zeit der Hauptlieferant für LSD in San Francisco war. Sheily Whiteley beschreibt die Londoner psychedelische Szene wie folgt:

»Long, improvisatory passages and electronically produced sound effects resonated with stroboscopic lighting to bring about a feeling of freedom analogous to the effect of acid: the spiling up of new sensations, < the associations with changed perspectives and color« (Whiteley 1992: 33).

In dieser Verbindung könnten die Konzerte als Beispiele für Komplementierung betrachtet werden, bei der Musik den akustischen Teil der synästhetischen LSD-Erfahrung repliziert oder stimuliert, während die Light Show die halluzinogene visuelle Dimension beisteuert. Performances mit visuellen und akustischen Medien vereinten sich im Psychedelic Rock-Konzert, um die vollständige Nachbildung eines LSD-Trips zu liefern, die keine von beiden für sich allein hätte produzieren können. ${ }^{10}$

Doch auch abgesehen von dem Kontext psychedelischer Drogenerfahrungen können psychedelische Light Shows als Beispiel für Komplementierung verstanden werden. Das Verhältnis der Headligths zu Jefferson Airplane war ungewöhnlich (zumindest für die USA). ${ }^{11}$ Normalerweise arbeiteten die

10 In diesem Kontext sei auch auf Whiteley (1992: 31-33) verwiesen, die die Frage aufgreift, ob Pink Floyds abgefahrenes »Astronomy Dominé« bereits an sich psychedelisch oder von dem Vorhandensein einer Light Show abhängig ist, um einen psychedelischen Effekt zu erschaffen. Nach Abwägen der Argumente schlussfolgert Whiteley, dass der Song rein auf der musikalischen Ebene psychedelisch ist.

11 Barry Miles (2004: 170) weist darauf hin, dass »the psychedelic light show developed differently in Britain than in the States. In America, the light-show teams operated independently, as if they were groups themselves, and would be hired to provide a show for all the groups playing that evening, whereas in 
Lichtkünstler für einen Veranstaltungsort und nicht für die Bands. Als feste Light Show am Fillmore East wurde die Joshua Light Show von Bill Graham engagiert, dem Impresario der beiden Spielstätten Fillmore East und Fillmore West, und nicht von den Bands, mit denen sie arbeiteten. Sie lieferten die Light Shows für alle, oftmals sehr verschiedenen Künstler, die Graham für die Hallen buchte (mit Ausnahme derer, die sich weigerten). Diese Light Shows waren normalerweise keine Zusammenarbeiten zwischen Lichtkünstlern und Musikern (obwohl die Lichtkünstler durchaus spezielle Wünsche der Musiker annahmen), und sie probten auch nicht zwangsläufig zusammen. White betont die improvisatorischen, die »manuellen « (gegenüber automatisierten) und die Live-Aspekte der Light Shows und deutet an, dass sich die Beteiligten meist wenig darum bemühten, eine enge Synchronisation zwischen Musik und Licht zu erreichen. Er beschreibt die Light Show als »arrhythmic and therefore it was the audience and the musicians which gave it a rhythm « (zit. n. Pouncey 2005b: 175). Die den Konzerten zugrunde liegende Annahme bestand darin, dass Musiker und Lichtkünstler eine Sensibilität, ein Gefühl für ihren gegenkulturellen Kontext und ein Bewusstsein dafür teilten, was ihr Publikum wollte. Sie alle arbeiteten für ein gemeinsames Ziel, jedoch nicht durch eine formale Zusammenarbeit oder die Anpassung von Klang und Visuellem.

Wenngleich Cook sein Schema für musikalische Multimedialität als ein quasi-objektives, strukturalistisches Vokabular zur Identifizierung unterschiedlicher Beziehungen zwischen den Elementen, die ein gegebenes IMM konstituieren, darstellt, bin ich der Meinung, dass die Auswahl (oder der Schwerpunkt auf) einer seiner Kategorien gegenüber einer anderen stärker die Ideologie, Vorurteile oder Interessen des Analysten widerspiegelt als die inhärenten Eigenschaften des betreffenden IMM.

Wie die von mir zitierten Beispiele andeuten, liegt es nahe, dass Musiker und Lichtkünstler, die an der Produktion psychedelischer Konzerte beteiligt waren, sowie ihr Publikum die Beziehung zwischen Musik und Light Show als Konformität oder Komplementierung oder beides ansahen. Vermutlich nahmen sie die Lichter als der Musik folgend oder sie illustrierend wahr oder sie verstanden Musik und Lichter als parallele Ereignisse (jedes der beiden ist ein Fall von Konformität). Da die Gegenkultur, von der Psychedelic Rock-

Britain any band wanting a light show tended to develop their own. "Das trifft indes nicht ganz zu, denn es gab eindeutig britische Light Shows, die unabhängig für mehrere Bands gearbeitet haben (vgl. Iles 2005: 79). Diese Tendenz mag teilweise erklären, warum Sheila Whiteley, die über die Londoner Szene schreibt, bekundet, dass die Light Shows dem Rhythmus der Musik folgten, während Joshua White über seine Arbeit in New York sagt, die Light Shows seien »arrhythmic« gewesen (beide sind im Haupttext zitiert). 
Konzerte ja ein Teil waren, synästhetische Erfahrungen betonte, könnten sie auch die auditiven und visuellen Medien als zusammenwirkend aufgefasst haben, um ein Gesamt-Event zu kreieren, unabhängig davon, ob die Veranstaltung als Simulation eines LSD-Trips oder als Zelebrieren gegenkultureller Ästhetiken verstanden wurde.

Aus der traditionalistischen Perspektive, die ich hier beschrieben habe, kann die Psychedelic Rock-Show jedoch nur als eine Form von Wettstreit zwischen den auditiven und visuellen Elementen des Konzerts verstanden werden. Diese Charakterisierung leitet sich nicht von der Beziehung zwischen den visuellen und den auditiven Elementen solch einer Show ab, sondern von der Beschaffenheit der visuellen Elemente selbst. Ein Traditionalist würde vermutlich keinen Einwand gegen eine Performance haben, in der die visuellen und musikalischen Elemente miteinander konform gehen oder einander komplementieren; in beiden Fällen könnten sie zusammen wirken, um musikalische Informationen zu kommunizieren, solange die betreffenden visuellen Elemente »musikalisch relevant « wären. In psychedelischen Light Shows jedoch waren die performenden Körper der Musiker in einer Art und Weise verflochten mit bewegten Bildern, Farben und Mustern, einige abstrakt, andere gegenständlich, die ihre Gesten und Gesichtsausdrücke verdeckten.

Die historische Wahrheit verlangt, die frühen Light Shows in San Francisco von den etwas späteren Praktiken an der Ostküste zu unterscheiden. In San Francisco Mitte der 1960er Jahre, wo musikalische Performances psychedelischer Rockbands eher als Tanzveranstaltungen denn als Konzerte inszeniert wurden, schufen die Light Shows suggestive visuelle Umgebungen, in die Musiker und Publikum gleichermaßen eintauchten. Spätere OstküstenLight Shows, besonders im Fillmore East, stützten sich eher auf Rückprojektionen auf Leinwänden hinter den Musikern, die sie weniger verdeckten. ${ }^{12}$ Im ersten Fall löschte die Light Show die Gesten und Gesichtsausdrücker der

12 Joshua White führt die Ursprünge der Ostküsten-Light Shows auf eine einwöchige Veranstaltung in Toronto im Jahre 1967 zurück, für die Bill Graham plante, die San Francisco-Szene nachzubilden. Der Veranstaltungsort war allerdings ein traditionelles Theater und kein offener Tanz- bzw. Festsaal wie das Fillmore Auditorium. Graham trat an White und seine Firma heran, um einen Weg zu finden, die Festsaal-Atmosphäre in einer konventionellen Theaterumgebung nachzubilden, was White durch den Einsatz von Rückprojektionen auf der Bühne und atmosphärischer Beleuchtung im Saal erreichte. Wenngleich White nicht die endgültige Light Show für diese Veranstaltung lieferte, war er doch an ihrer Entwicklung beteiligt. Letztlich war es die Adaption der Techniken und Bildsprache der San Francisco-Light Show an eine Theaterumgebung durch den Gebrauch von Rückprojektionen, die die Geburt der Ostküsten-Light Shows bedeutete (vgl. Pouncey 2005b: 167-169 und Del Signore 2007). 
Musiker aus; im letzteren Fall bot die Light Show eine verwirrend spektakuläre Konkurrenz für die Musiker. Der Effekt der Ablenkung war beabsichtigt. Joshua White schreibt seine Chance, am Fillmore East Light Shows zu entwerfen, Bill Grahams Entscheidung zu, »that the audience needed to have something to look at besides a bunch of musicians in street clothes tuning up « (Bill Graham zit. in Del Signore 2007). In beiden Fällen, selbst wenn die visuellen Effekte dem Rhythmus oder der Struktur der Musik folgten und dadurch musikalische Informationen an das Publikum übertrugen, bedrohte die Light Show doch unweigerlich die Möglichkeit des Publikums, ebenjene Dinge zu sehen oder zu fokussieren, die im traditionalistischen Modell der musikalischen Performance im Mittelpunkt stehen: die physischen Handlungen des Musikers zur Klangproduktion. Das Publikum wurde so dessen beraubt, was es nach Ansicht der Traditionalisten braucht, um Einsatz und Fähigkeiten des Musikers wahrzunehmen und die Authentizität der Performance zu verifizieren. Die Light Show machte sich desselben Vergehens schuldig, dessen Traditionalisten gelegentlich Musikvideos bezichtigen:

»substituting the gestures of a performer with other visual content necessarily changes perceptual and affective co-regulation, distorting and diluting the communication between performers and listeners through a literal distancing of the performer from his or her audience" (Thompson/Graham/Russo 2005: 222).

Auch wenn ich das Rockkonzert mit psychedelischer Light Show als eine Performance-Praxis bezeichne, die die traditionalistische Sichtweise der musikalischen Performance und die ihr innewohnenden Werte herausforderte, ist es schwierig, mit Sicherheit zu bestimmen, ob psychedelische Rockbands wie Jefferson Airplane den eigenen Einsatz von Light Shows als eine Herausforderung solch einer Sicht, wie die musikalische Performance zu sein habe, verstanden haben oder ob sie einfach den Performance-Konventionen ihres Genres, sozialen Milieus und historischen Zeitpunkts verhaftet waren. Es gibt allerdings Belege für einige Differenzen um den Rückgriff auf visuelle Spektakel im Psychedelic Rock. Chuck Beale, Leadgitarrist der kanadischen Band The Paupers, wird wie folgt zitiert:

$»$ We are trying to create a total environment with sound alone [...]. Sound is enough. We don't use lights or any gimmicks. When we record we don't double track or use any other instruments. What the four of us can do is the sound we make. That's all« (zit. n. Lydon 2003: 26).

Diese Äußerung zielte wahrscheinlich auf Jefferson Airplane, die angeblich von den Paupers an die Wand gespielt wurden, als beide Bands 1967 am 
selben Abend im Café Au Go Go in New York auftraten. Sie deutet darauf hin, dass die Gemeinschaft der Psychedelic Rock-Musiker nicht monolithisch war, sondern dass es verschiedene Lager gab, einschließlich eines traditionalistischen Lagers, das »musikalisch irrelevante « Visualisierungen nebst Trickserei im Aufnahmestudio ablehnte, zugunsten der Fokussierung auf die Musiker, ihren Sound und ihre ungeschönten Fähigkeiten, diesen hervorzubringen.

\section{Fazit}

Es ist klar, dass technologische Veränderungen den Hintergrund für die audiovisuelle Ökonomie der musikalischen Performance darstellen, die ich hier beschrieben habe. Technologisch induzierte Entwicklungen, die die traditionalistischen Werte herausforderten, welche die Wichtigkeit der Aufrechterhaltung klarer Ursache/Wirkung-Beziehungen zwischen den visuellen und auditiven Aspekten der musikalischen Performance unterstreichen, schließen Tonaufnahmen, Rundfunk und den Einsatz von Computern und digitalen Instrumenten bei Liveauftritten ein, ebenso wie jüngere kulturelle Formen wie das Musikvideo und die Luftgitarre. Alles in allem weisen die Belege darauf hin, dass die traditionalistische Angst vor diesen Entwicklungen unangebracht ist. Sosehr die Ästhetiken aktueller Performances populärer Musik auch von rein visuellen Spektakeln dominiert zu sein scheinen, so bleiben sie ideologisch doch der traditionalistischen Denkweise verhaftet, dass wir eine visuelle Überprüfung der musikalischen Klänge benötigen. Wenn neue Wege des Musikmachens aufkommen, finden seine Praktiker in den meisten Fällen Wege, sie traditionalistischen Werten anzupassen. Dies wird z.B. an der Debatte um Computermusik sichtbar, mit der ich begonnen habe. Als weitere Ausgestaltung dieses Beispiels möchte ich eine EmailEinladung zu einem Abend mit live kodierter Musik im Anatomy Museum, King's College, London, zitieren, die mich am 9. Januar 2010 erreichte. Dort heißt es: "Live coders expose and rewire the innards of software while it generates improvised music and/or visuals« (»live coding « wird auch »onthe-fly programming « genannt; Princeton University Computer Science 2002). Im weiteren Text wird uns versichert: »All code manipulation is projected for your pleasure.« Es wird deutlich, dass sich die traditionellen Annahmen selbst in diesem exklusiven, technologisch fortgeschrittenen musikalischen Kontext behaupten: dem Publikum muss, egal auf welche Weise, gezeigt werden, wie der Klang gemacht wird. 
Ein ganz ähnlicher Fall ereignete sich kürzlich bei einem Auftritt des Jazzgitarristen Pat Metheny mit dem von ihm entwickelten Orchestrion, einer komplexen Anordnung robotisierter musikalischer Instrumente, vornehmlich Saiten- und Percussioninstrumente. Das Orchestrion wird von einem Computer und einem MIDI-Interface gesteuert und begleitet sein Gitarrenspiel. Bei diesen Konzerten ist Metheny der einzige »menschliche« Performer auf der Bühne.

Ben Ratliffs Besprechung von Methenys Orchestrion-Konzert 2010 in der Town Hall in New York City zeichnet interessanterweise nach, wie das Konzert strukturiert gewesen sein muss, um einem von Traditionalismus durchdrungenen Publikum deutlich zu machen, was dort geschah. Ratliff beginnt mit der Bemerkung, dass Metheny zunächst Sologitarre spielte, etwas, an das sein Publikum gewöhnt war und in dem die visuellen Beziehungen von Ursache und Wirkung zwischen dem, was er tat, und den Klängen, die er produzierte, klar war. Ratliff nimmt an, das Publikum hätte revoltiert, wenn Metheny ihm keine Verständnishilfen gegeben hätte. Über den folgenden Abschnitt des Konzerts schreibt er:

»It seems that the specificity of your attack on the guitar - whether and how you strum a chord or pick a note - determines the texture of the orchestral sounds that result from it. How it all works remains unclear, but the audience understands it better. [...] It's quite possible that a listener is thinking, for the first time that evening, >I could do that « (Ratliff 2010).

Ratliffs Beschreibung lässt den Schluss zu, dass Metheney, um seine Performance einer ungewohnten Musik einem traditionalistischen Publikum schmackhaft zu machen, dem weiter oben zitierten Vorschlag von Schloss folgt, indem er deutlich sichtbare Eingabeparameter bzw. -gesten präsentiert, um die Beziehung zwischen Geste und Klang zu verdeutlichen und das Publikum glauben zu machen, es verstünde, was gerade vorgeht.

Obwohl es keinen Grund gibt anzunehmen, dass die traditionalistischen Werte für die musikalische Performance in naher Zukunft ihre Vorherrschaft verlieren, zeigt die Berücksichtigung der historisch uneinheitlichen Beziehung zwischen Klang und Visuellem in der musikalischen Performance, dass das »Audiovisuelle « in diesem Kontext nicht als nahtlose Einheit zu verstehen ist, sondern als synkretistisch. Wahrscheinlich ist es besser als »audio-visuell« oder »audio/visuell « zu bezeichnen. In den kulturellen Kontexten von Rock, Jazz und Computermusik, auf die ich mich hier bezogen habe, ist das Audiovisuelle immer in zwei Tracks unterteilt, die als eigenständig behandelt und in diverse Beziehungen zueinander gesetzt werden können. Diese Beziehungen sind hierarchisierend konstruiert, ein Track wird 
immer als dominierend und den Kontext bestimmend gedacht. Diese traditionalistische Position, die die dominante bleibt, hat eine komplexe Beziehung zwischen diesen Tracks zur Folge. Einerseits wird der Klang als der bedeutendere Track angesehen, weil das Ziel der Performance eben das Spielen der Musik ist. Andererseits ist der Klang allein nicht dazu im Stande, das Publikum mit allen notwendigen Informationen zu versorgen, um die Fähigkeiten und Anstrengungen des Musikers beurteilen zu können; es bleibt zur Überprüfung vom visuellen Track abhängig. In diesem Sinne ist die Vorherrschaft im visuellen Track verortet. Es ist dieser Track, den Musiker, die die traditionalistischen Werte und Performance-Konventionen herausfordern wollten, manipulierten, indem sie Lichteffekte, einschließlich Dunkelheit und psychedelischer Light Shows, verwendeten, so dass ihre eigenen Aktionen während der musikalischen Klangerzeugung verschleiert wurden und spektakuläre visuelle Effekte ihre Performances dominierten.

\section{Literatur}

Auslander, Philip (2006). Performing Glam Rock: Gender and Theatricality in Popular Music. Ann Arbor: University of Michigan Press.

Auslander, Philip (2008). Liveness: Performance in a Mediatized Culture. London: Routledge (2. Aufl.).

Butler, Mark J. (o.J.). Playing with Something that Runs: Technology, Improvisation, and Composition in Electronic-Music Performance. Unveröffentlichtes Typoskript.

Cook, Nicholas (2001). Analysing Musical Multimedia. Oxford, New York: Oxford University Press.

Del Signore, John (2007). »Joshua White, >The Joshua Light Show «.« In: The Gothamist vom 2. April; http://gothamist.com/2007/04/02/interview_joshu.php (Zugriff: 30.8.2012).

Dickinson, Kay (2007). »Music Video and Synaesthetic Possibility. «In: Medium Cool. Hg. v. Roger Beebe und Jason Middleton. Durham, NC: Duke University Press, S. 13-29.

Godlovitch, Stan (1998). Musical Performance: A Philosophical Study. London, New York: Routledge.

Gracyk, Theodore (1997). »Listening to Music: Performances and Recordings. «In: The Journal of Aesthetics and Art Criticism 55, S. 139-150.

Grunenberg, Christoph (2005). "The Politics of Ecstasy: Art for the Mind and Body.« In: Summer of Love: Art of the Psychedelic Era. Hg. v. Christoph Grunenberg. Liverpool: Tate Liverpool, S. 11-43.

Hamlin, Jesse (1999). »Painting Live with Light and Music. In: San Francisco Chronicle vom 31. Januar; http://articles.sfgate.com/1999-01-31/entertainment/ 17677751_1_mckay-s-work-paintings-mckay-s-head-lights (Zugriff: 30.10.2012).

Iles, Chrissie (2005). »Liquid Dreams. «In: Summer of Love: Art of the Psychedelic Era. Hg. v. Christoph Grunenberg. Liverpool: Tate Liverpool, S. 67-83. 
Jay, Martin (1994). Downcast Eyes: The Denigration of Vision in Twentieth Century French Thought. Berkeley, Los Angeles, London: The University of California Press.

Laing, Dave (1991). »A Voice without a Face: Popular Music and the Phonograph in the 1890s. «In: Popular Music 10, Nr. 1, S. 1-9.

Lydon, Michael (2003). Flashbacks: Eyewitness Accounts of the Rock Revolution 1964-1974. New York, London: Routledge.

Miles, Barry (2004). Hippie. New York: Sterling Publishing.

Pouncey, Edwin (2005a). "Laboratories of Light: Psychedelic Light Shows. «In: Summer of Love: Psychedelic Art, Social Crisis and Counterculture in the 1960s. Hg. v. Christopher Grunenberg und Jonathan Harris. Liverpool: Liverpool University Press and Tate Liverpool, S. 155-162.

Pouncey, Edwin (2005b). »I Never Stopped Loving the Light: Joshua White and the Joshua Light Show. «In: Summer of Love: Psychedelic Art, Social Crisis and Counterculture in the 1960s. Hg. v. Christopher Grunenberg und Jonathan Harris. Liverpool: Liverpool University Press and Tate Liverpool, S. 163-178.

Princeton University Computer Science (2002). »On-the-fly Programming: Using Code as an Expressive Musical Instrument. Princeton: Computer Science; http://on-the-fly.cs.princeton.edu (Zugriff: 30.8.2012).

Ratliff, Ben (2010). »If Not 76 Trombones, Everything Else a One-Man Band Can Handle. «In: New York Times vom 23. Mai; http://www.nytimes.com/2010/05/ 24/arts/music/24metheny.html (Zugriff: 30.8.2012).

Schloss, W. Andrew (2003). »Using Contemporary Technology in Live Performance: The Dilemma of the Performer.«In: Journal of New Music Research 32, Nr. 3, S. 239-242.

Schutz, Michael (2008). "Seeing Music? What Musicians Need to Know about Vision. « In: Empirical Musicology Review 3, Nr. 3, S. 83-108; http://hdl.handle.net/1811/ 34098 (Zugriff: 30.8 .2012 ).

Sterne, Jonathan (2003). The Audible Past: Cultural Origins of Sound Reproduction. Durham, NC: Duke University Press.

Stuart, Caleb (2003). »The Object of Performance: Aural Performativity in Contemporary Laptop Music. «In: Contemporary Music Review 22, Nr. 4, S. 59-65.

Tamarkin, Jeff (2003). Got A Revolution: The Turbulent Flight of Jefferson Airplane. New York: Atria Books, 2003.

Thompson, William Forde / Graham, Phil / Russo, Frank A. (2005). "Seeing Musical Performance: Visual Influences on Perception and Experience. «In: Semiotica 156, S. 203-227.

University of Victoria (o.J.). »W. Andrew Schloss.« In: University of Victoria, School of Music, Fine Arts; http://finearts.uvic.ca/music/ contacts /faculty/Bios/ aschloss.shtml (Zugriff: 30.8.2012).

Whiteley, Sheila (1992). The Space Between the Notes: Rock and the CounterCulture. London, New York: Routledge.

Zinman, Gregory (2008). »The Joshua Light Show: Concrete Practices and Ephemeral Effects. «In: American Art 22, Nr. 2, S. 17-21. 


\title{
Disko- und Videographie
}

Fox, Virgil (o.J.). »Virgil Fox Heavy Organ«; http://www.youtube.com/watch?v= gIPCX3Te-BA (Zugriff: 30.8.2012).

Jefferson Airplane (1969). »Turn Out The Lights. « Auf: Bless Its Pointed Little Head. LP. RCA Records LSP-4133.

\begin{abstract}
By characterizing the relationship between the visual and audible dimensions of music performance as an »economy«, I am suggesting that these two dimensions do not necessarily simply work hand-in-hand. Rather, there can be competition between them as they vie for the audience's attention and seek to influence the audience's understanding of the performance. Musicians shape differential relationships between sight and sound some of which can be characterized as normative or traditional, while others can be characterized as challenging the norms.

I begin by positing what I call the straditionalist « view of music performance, a view that emphasizes visible causality in music performance: what the audience sees should provide information about how the sound is being produced and, perhaps, about the musician's affective state. Visual information that does not contribute to this understanding is perceived as interference. The relative value of sound and visual information in music performance varies somewhat by genre, of course. But even performers operating within genre contexts in which the traditional values generally hold sway sometimes challenge those values by manipulating the relationship between the auditory and visual aspects of musical performance in ways that go against the traditional (or traditionalist) grain. In my essay, I look at one such performance practice: the use of light shows in psychedelic rock concerts of the late 1960s and early 1970s.
\end{abstract}

